\title{
A STONE-WEIERSTRASS THEOREM WITHOUT CLOSURE UNDER SUPREMA
}

\author{
R. PRESTON M $^{\mathrm{c}}$ AFEE AND PHILIP J. RENY
}

(Communicated by Palle E. T. Jorgensen)

\begin{abstract}
For a compact metric space $X$, consider a linear subspace $A$ of $C(X)$ containing the constant functions. One version of the Stone-Weierstrass Theorem states that, if $A$ separates points, then the closure of $A$ under both minima and maxima is dense in $C(X)$. By the Hahn-Banach Theorem, if $A$ separates probability measures, $A$ is dense in $C(X)$. It is shown that if $A$ separates points from probability measures, then the closure of $A$ under minima is dense in $C(X)$. This theorem has applications in economic theory.
\end{abstract}

The classical Stone-Weierstrass Theorem states that, if a linear space $A$ of real valued functions defined on a compact metric space $X$ contains the constant functions, is closed under minima and maxima, and separates points, then $A$ is dense in $C(X)$. The purpose of this paper is to provide an alternative structure for sets closed under minima alone, which generates the same result.

The theorem fits between the Stone-Weierstrass Theorem and a corollary to the Hahn-Banach Theorem. Let $X$ be a compact metric space, with metric $\rho$, and $\Delta$ the set of probability measures (regular unitary measures) on $X$. Let $\delta_{x}$ represent the point mass measures:

$$
\delta_{x}(E)= \begin{cases}1, & \text { if } x \in E, \\ 0, & \text { if } x \notin E .\end{cases}
$$

For $A \subseteq C(X)$, define the closure under minima and maxima:

$$
\begin{aligned}
& A_{m}=\left\{f: f(x)=\min _{1 \leq i \leq n} f_{i}(x), f_{i} \in A, n \in \mathbf{N}\right\}, \\
& A_{M}=\left\{f: f(x)=\max _{1 \leq i \leq n} f_{i}(x), f_{i} \in A, n \in \mathbf{N}\right\} .
\end{aligned}
$$

As usual, 1 denotes the constant function one, and $\bar{A}$ the closure of $A$ in supnorm.

Definition 1. A linear subspace of $C(X)$ containing 1 is said to separate points

Received by the editors April 6, 1990.

1991 Mathematics Subject Classification. Primary 46B28, 26A15.

The authors gratefully acknowledge the assistance of Charalambos Aliprantis in the preparation of this paper. 
if, for $x$ and $y$ in $X$,

$$
\int f d \delta_{x}=\int f d \delta_{y} \quad \text { for all } f \text { in } A \text { implies } \quad x=y,
$$

and to separate probability measures if, for $\mu, \nu \in \Delta$,

$$
\int f d \mu=\int f d \nu \text { for all } f \text { in } A \text { implies } \quad \mu=\nu,
$$

and to separate points from probability measures if, for $\mu$ in $\Delta, x \in X$,

$$
\int f d \mu=\int f d \delta_{x} \quad \text { for all } f \text { in } A \text { implies } \quad \mu=\delta_{x} .
$$

One statement of the Stone-Weierstrass Theorem is

Theorem 2 (Stone-Weierstrass). If $A$ is a linear subspace of $C(X), \mathbf{1} \in A$, then $A$ separates points if and only if $\overline{\left(A_{m}\right)_{M}}=C(\mathbf{X})$.

Condition (1) is equivalent to the more standard definition of separating points, namely that $f(x)=f(y)$ for all $f \in A$ implies $x=y$, and is stated in the somewhat cumbersome manner above for comparability to two subsequent results. Note that $\left(A_{m}\right)_{M}$ is a linear space closed under maxima and minima.

A well-known corollary ${ }^{1}$ to the Hahn-Banach Theorem and Riesz Representation Theorem has a similar flavor to Theorem 2 .

Theorem 3 (Corollary to Hahn-Banach). If $A$ is a linear subspace of $C(X)$, $\mathbf{1} \in A$, then $A$ separates probability measures if and only if $\bar{A}=C(X)$.

Thus, one consolidated view of these results is that we are given $A \subseteq C(X)$, with $1 \in A$, then $A$ is dense if it separates probability measures from probability measures, or if it is closed under minima and maxima and separates points. In the next section, we prove the following intermediate result.

Theorem 4. If $A$ is a linear subspace of $C(X), \mathbf{1} \in A$, then $A$ separates points from probability measures if and only if $\overline{A_{m}}=\overline{A_{M}}=C(X)$.

This demonstrates that separating points from probability measures substitutes for the ability to take maxima in the Stone-Weierstrass Theorem.

Consider for example, the set of quadratics on $[0,1]$ :

$$
A=\left\{\alpha_{0}+\alpha_{1} x+\alpha_{2} x^{2}:\left(\alpha_{0}, \alpha_{1}, \alpha_{2}\right) \in \mathbf{R}^{3}\right\} .
$$

Clearly $\bar{A}=A \neq C[0,1]$. However, $A$ separates points from probability measures. That is if $\mu \in \Delta, \mu \neq \delta_{y}$, then

$$
\int(x-y)^{2} d \delta_{y}(x)=0<\int(x-y)^{2} d \mu(x) .
$$

Therefore, according to Theorem $4, \overline{A_{m}}=\overline{A_{M}}=C[0,1]$. Thus, the present theorem is not a consequence of the Hahn-Banach Corollary. Similarly, if $A$ is the set of linear functions on $[0,1]$,

$$
A=\left\{\alpha_{0}+\alpha_{1} x:\left(\alpha_{0}, \alpha_{1}\right) \in \mathbf{R}^{2}\right\} .
$$

\footnotetext{
${ }^{1}$ See, for example, Friedman [3, Corollary 4.8.7, p. 153], and note that the norm dual of $C(X)$ is the set of regular signed measures. Since $1 \in A, \int f\left(d \mu^{+}-d \mu^{-}\right)=0$ allows $\mu^{+}, \mu^{-} \in \Delta$ without loss of generality, where $\mu=\mu^{+}-\mu^{-}$is the Jordan decomposition of $\mu$.
} 
Since $A$ separates points, $\overline{\left(A_{m}\right)_{M}}=C[0,1]$. Incidentally, $\left(A_{m}\right)_{M}$ is the subspace of all piecewise linear functions on $[0,1]$. However, $A$ fails to separate points from probability measures, and $\overline{A_{m}}$ is only the set of convex functions. This example distinguishes the present theorem from the Stone-Weierstrass theorem.

Problems for which only minima or maxima, but not both, may be taken arise in a natural way in economic theory. Suppose the value $\nu$ of an object for sale (e.g., an oil lease) is correlated to an observable $s$ (for example, the results of a sample drilling). Let $f(s / \nu)$ be the density of $s$, given $\nu$. Suppose the potential buyer, but not the seller, knows $\nu$. Can the seller on average charge the potential buyer his value $\nu$ ? This reduces to solving the equation

$$
v=\int_{S} z(s) f(s / \nu) d s,
$$

where $z(s)$ is the price charged when the outcome $s$ arises. Assume $s$ is a draw from a compact metric space $S$.

If the seller offers the buyer a set $\left\{z_{1}, \ldots, z_{n}\right\}$ of price functions, and lets the buyer choose the one he likes best (i.e., which minimizes the expected price) the seller will learn, on average,

$$
p(\nu)=\min _{1 \leq i \leq n} \int_{S} z_{i}(s) f(s / \nu) d s .
$$

This requires that $p(\nu)$ be no greater than the buyer's value, $\nu$, so that the buyer is willing to participate in this scheme.

$$
R=\left\{\int z(s) f(s / \cdot) d s: z \in C(S)\right\},
$$

then the seller can charge the buyer his value (on average) precisely when the identity is in $R_{m}$. Obviously, the seller can get arbitrarily close if $\overline{R_{m}}=$ $C[0, \bar{\nu}]$, where the value falls in $[0, \bar{\nu}]$. Note that $1 \in R$ since $f(\cdot / \nu)$ is a density. This problem, and others like it, are explored in $[4,5]$. We shall return to a special case of this class of problems in the final section.

\section{Proof OF THEOREM 4}

For this section, $X$ is a compact metric space with metric $\rho, A$ is a linear subspace of $C(X)$, and $\mathbf{1} \in A$.

Definition 5. Let $\varepsilon>0, \delta>0$. A positive continuous function $f$ is a nearly $u$-shaped function at $y$ of order $(\varepsilon, \delta)$ if (i) $f(y) \leq \varepsilon$ and (ii) $\rho(x, y)>\delta$ implies $f(x) \geq 1$.

Let $U(y, \varepsilon, \delta)$ denote the set of nearly $u$-shaped functions at $y$ of order $(\varepsilon, \delta)$. We shall make use of three obvious properties of the sets $U(y, \varepsilon, \delta)$.

$$
\begin{gathered}
0<\varepsilon \leq \varepsilon_{0}, 0<\delta \leq \delta_{0} \Rightarrow U(y, \varepsilon, \delta) \subseteq U\left(y, \varepsilon_{0}, \delta_{0}\right), \\
\quad \text { each } U(y, \varepsilon, \delta) \text { is convex, } \\
\text { each } U(y, \varepsilon, \delta) \text { has nonempty interior. }
\end{gathered}
$$

The last fact follows from the observation that the $\varepsilon / 4$ ball around $\varepsilon / 2+$ $\rho(\cdot, y) / \delta$ is contained in $U(y, \varepsilon, \delta)$. The following lemma shows that $\overline{A_{m}}=$ $C(X)$ if and only if $A$ contains nearly $u$-shaped functions at every $x \in X$ of all orders $(\varepsilon, \delta)$. This lemma is critical to the proof of the theorem. 
Lemma 6. Suppose $A \subseteq C(X)$ is a linear subspace, $\mathbf{1} \in A$. Then $\overline{A_{m}}=C(X)$ if and only if for all $y$ in $X$ and all $\varepsilon, \delta>0, U(y, \varepsilon, \delta) \cap A \neq \varnothing$.

Proof. $(\Rightarrow)$ Fix $y \in X, \varepsilon>0$, and $\delta>0$. Since $\overline{A_{m}}=C(X)$, there are $f_{i}, \ldots, f_{n} \in A$ so that

$$
\left|\min _{1 \leq i \leq n} f_{i}(x)-(\varepsilon / 2+\rho(x, y) / \delta)\right|<\varepsilon / 2, \quad \forall x \in X .
$$

Thus, there exists $j \in\{1, \ldots, n\}$ with $\left|f_{j}(y)-\varepsilon / 2\right|<\varepsilon / 2$. From $f_{j}(x) \geq$ $\min _{1 \leq i \leq n} f_{i}(x) \geq \rho(x, y) / \delta$, we easily infer $f_{j} \in U(y, \varepsilon, \delta)$.

$(\stackrel{\Leftarrow}{\Leftarrow})$ Fix $f \in C(X)$ and $\varepsilon>0$. Define

$$
\alpha=\max _{x \in X} f(x)-\min _{x \in \mathbf{X}} f(x) .
$$

If $\alpha=0$, we are done, since $1 \in A$. So suppose $\alpha>0$. Since $f$ is continuous and $X$ compact, there is a $\beta>0$ so that

$$
\rho(x, y)<\beta \Rightarrow|f(y)-f(x)|<\varepsilon / 2 .
$$

For each $y \in X$, choose $g \in A \cap U(y, \varepsilon / 3 \alpha, \beta)$, and define

$$
h=\alpha g+(f(y)+\varepsilon / 2) \mathbf{1} \in A \text {. }
$$

Note

$$
|h(y)-f(y)|=\alpha g(y)+\varepsilon / 2 \leq \alpha(\varepsilon / 3 \alpha)+\varepsilon / 2<\varepsilon .
$$

For $\rho(x, y)<\beta$,

$$
h(x)-f(x)=\alpha g(x)+f(y)-f(x)+\varepsilon / 2 \geq f(y)-f(x)+\varepsilon / 2 \geq 0 .
$$

For $\rho(x, y) \geq \beta$, we have

$$
h(x)-f(x)=\alpha g(x)+f(y)-f(x)+\varepsilon / 2 \geq \alpha+f(y)-f(x)+\varepsilon / 2 \geq 0,
$$

by the definition of $\alpha$.

Thus $h(x) \geq f(x)$ and $h(y)<f(y)+\varepsilon$. Now define the set (recall that $h$ depends on $y)$ :

$$
S(y)=\{x: h(x)<f(x)+\varepsilon\} .
$$

Clearly, $\{S(y): y \in X\}$ forms an open cover of $X$, since $y \in S(y)$. Because $X$ is compact, there is a finite subcover $S\left(x_{1}\right), \ldots, S\left(x_{n}\right)$, with associated functions $h_{1}, \ldots, h_{n}$. By construction, $0 \leq \min _{1 \leq i \leq n} h_{i}(x)-f(x)<\varepsilon$ for all $x \in X$, and thus $f \in \overline{A_{m}}$ as desired. Q.E.D.

Remark. The nearly $u$-shaped functions permit approximation from above, in the sense that the lower envelope, produced by minima, approximates any function. This occurs because nearly $u$-shaped functions take minima near a chosen point $y$, and then rise sufficiently rapidly away from $y$.

Theorem 4. Suppose $A$ is a linear subspace of $C(X)$, where $X$ is a compact metric space, and $\mathbf{1} \in A$. Then $\overline{A_{m}}=C(X)$ if and only if $A$ separates points from probability distributions.

Proof. $(\Rightarrow)$ Suppose $\mu \in \Delta, \mu \neq \delta_{y}$. Then there exists $\delta>0$ so that $\int_{N_{\delta}(y)} d \mu(x)<1$, where $N_{\delta}(y)=\{x: \rho(x, y)<\delta\}$. Let $\varepsilon<1-\int_{N_{\delta}(y)} d \mu(x)$, 
and choose $f \in U(y, \varepsilon, \delta) \cap A$. Such a function exists by Lemma 6 . Then

$$
\begin{aligned}
\int f d \mu & =\int_{N_{\delta}(y)} f d \mu+\int_{X \backslash N_{\delta}(y)} f d \mu \geq \int_{X \backslash N_{\delta}(y)} f d \mu \geq \int_{X \backslash N_{\delta}(y)} d \mu \\
& =1-\int_{N_{\delta}(y)} d \mu>\varepsilon \geq f(y),
\end{aligned}
$$

and so $f \in A$ and $f$ separates $\delta y$ from $\mu$, as desired.

$(\Leftrightarrow)$ Suppose, by way of contradiction, that $\overline{A_{m}} \neq C(X)$. By Lemma 6 , there exists $y, \varepsilon_{0}>0$, and $\delta_{0}>0$ so that $U\left(y, \varepsilon_{0}, \delta_{0}\right) \cap A=\varnothing$. Since $A$ is linear, and hence convex, and $U\left(y, \varepsilon_{0}, \delta_{0}\right)$ is convex, with nonempty interior, there is a separating functional ${ }^{2}$ Thus, there is a nonzero signed measure $\mu$, and a constant $c$ satisfying

(6) for all $g \in A$ and all $f \in U\left(y, \varepsilon_{0}, \delta_{0}\right)$, we have $\int g d \mu \leq c \leq \int f d \mu$.

Since $A$ is a linear space, $\int g d \mu=0$ for all $g$ in $A$. Therefore, $c \geq 0$.

Let $\mu=\mu^{+}-\mu^{-}$be the Jordan Decomposition of $\mu$ (see [6, pp. 235-236]), with associated sets $S^{+}$and $S^{-}$, which partition $X$, satisfying

$$
S^{+} \cap S^{-}=\varnothing \text { and } \mu^{+}\left(S^{-}\right)=\mu^{-}\left(S^{+}\right)=0 .
$$

Since $1 \in A, \int d \mu^{+}=\int d \mu^{-}$.

Thus both $\mu^{+}$and $\mu^{-}$are finite, and we may then take $\mu^{+}, \mu^{-} \in \Delta$ without loss of generality, by rescaling. Neither $\mu^{+}$nor $\mu^{-}$can be $\delta_{y}$, for if either is equal to $\delta_{y},(2)$ and $\mu \neq 0$ contradicts (6). Since $\mu^{-}$is regular (see [1, Theorem 1.1, p. 7]), there is a closed set $\Psi \subseteq S^{-}$and $0<\delta \leq \delta_{0}$ so that $\Psi \cap N_{\delta}(y)=\varnothing$ and $\mu^{-}(\Psi)>0$. Choose $K>1 / \mu^{-}(\Psi) \geq 1$, and define

$$
f(x)= \begin{cases}0, & \text { if } x \in N_{\delta}(y), \\ K, & \text { if } x \in \Psi, \\ 1, & \text { if } x \notin \Psi \cup N_{\delta}(y),\end{cases}
$$

and observe, since $\Psi \cap S^{+}=\varnothing$, that

$$
\int f d \mu=\int f d \mu^{+}-\int f d \mu^{-} \leq 1-K \int_{\Psi} d \mu^{-}<0 .
$$

By $[1 \text {, Theorem 1.2, p. } 8]^{3}$ there is a sequence $\left\{f_{n}\right\} \subseteq C(X)$ satisfying

(a) $f_{n}(x) \geq 1$ for all $x \notin N_{\delta}\left(x_{0}\right)$;

(b) $0 \leq f_{n}(x) \leq K$ for all $x \in X$;

(c) $f_{n}(y)=0$; and

(d) $f_{n}(x) \rightarrow f(x)$ for all $x \in X$.

By (a)-(c), $f_{n} \in U\left(y, \varepsilon_{0}, \delta\right) \subseteq U\left(y, \varepsilon_{0}, \delta_{0}\right)$. By (b), (d), and (7), $\int f_{n} d \mu \rightarrow$ $\int f d \mu<0$, which contradicts (6) and $c \geq 0$. This completes the proof. Q.E.D.

Remark. $\overline{A_{m}}$ may be replaced by $\overline{A_{M}}$ in the statement of the theorem, by noting $A_{M}=-\left((-A)_{m}\right)=-A_{m}$, since $A$ is linear. In addition, if $A$ is a

\footnotetext{
${ }^{2}$ See [2, Part I, Theorem 8, p. 417].

${ }^{3}$ This is a straightforward application of the Tietze Extension Theorem.
} 
convex cone and both 1 and -1 are in $A$, Lemma 6 and Theorem 4 continue to hold, with trivial modifications of the proof.

\section{CONCLUSION}

When the metric space $X$ is an interval $[a, b]$ of the real line, the StoneWeierstrass Theorem has an appealing corollary, namely that if $\mathbf{1}$ and a strictly increasing function are in $A$, then $\overline{\left(A_{m}\right)_{M}}=C[a, b]$. There is an analogous corollary for the present theorem.

Corollary 7. Suppose $A$ is a linear subspace of $C[a, b]$ containing 1 and two functions $f$ and $g$ satisfying

$f$ is strictly increasing,

$$
\frac{g(x)-g(y)}{f(x)-f(y)} \text { is strictly increasing in } x \neq y, \quad \text { for all } y .^{4}
$$

Then $\overline{A_{m}}=C[a, b]$.

Proof. Observe that, if $x<y<z$, then

$$
\frac{g(x)-g(y)}{f(x)-f(y)} \leq \frac{g(z)-g(y)}{f(z)-f(y)} .
$$

Therefore, there is a function $\alpha$ (not necessarily continuous) so that

$$
\lim _{x \rightarrow y^{-}} \frac{g(x)-g(y)}{f(x)-f(y)} \leq \alpha(y) \leq \lim _{x \rightarrow y^{+}} \frac{g(x)-g(y)}{f(x)-f(y)} .
$$

Moreover, $\alpha$ is strictly increasing, for if $x<y<z$,

$$
\alpha(x)<\frac{g(y)-g(x)}{f(y)-f(x)}<\frac{g(y)-g(z)}{f(y)-f(z)}<\alpha(z) .
$$

Consider $\beta_{x}(y)=g(y)-\alpha(x) f(y)$, and note $\beta_{x}$ is in $A$ and satisfies

$$
\beta_{x}(y)-\beta_{x}(x)=(f(y)-f(x))\left[\frac{g(y)-g(x)}{f(y)-f(x)}-\alpha(x)\right] \geq 0,
$$

with equality if and only if $y=x$. Thus, if $\nu \neq \delta_{x}$,

$$
\int_{a}^{b} \beta_{x}(y) d \nu(y)>\int_{a}^{b} \beta_{x}(x) d \nu(y)=\beta_{x}(x) .
$$

Consequently, (2) is satisfied. Q.E.D.

Remark. If $f$ and $g$ are twice differentiable, (8) and (9) reduce to $f^{\prime}>0$ and $\left(g^{\prime} / f^{\prime}\right)^{\prime}>0$, which are easy to check, as we illustrate in the following example.

Example 7. Suppose a random variable $s$ has cumulative distribution function $s^{\nu}$ for $s \in[0,1]$. An economic agent who knows $\nu$ is to be offered a menu $\left\{z_{i}\right\}$ of payments. This agent chooses the charge with the least expected value:

$$
p(\nu)=\min _{1 \leq i \leq n} \int_{0}^{1} z_{i}(s) \nu s^{\nu-1} d s .
$$

\footnotetext{
${ }^{4}$ The function $(g(x)-g(y)) /(f(x)-f(y))$ necessarily has left and right limits as $x \rightarrow y$, if (9) holds, for $a<y<b$.
} 
Is the set of such charges dense in $C[0,1]$ ? That is, if the agent's value of an object for sale is $\pi(\nu)$, is there a menu $\left\{z_{i}(s)\right\}$ that approximately charges the agent his value?

The answer is yes. Consider

$$
A=\left\{f: f(\nu)=\int_{0}^{1} z(s) \nu s^{\nu-1} d s, \quad z \in C[0,1]\right\} .
$$

Note that $A$ contains 1 (using $z=1), f(\nu)=\nu /(\nu+1)$ (for $z(s)=s)$ ), and $g(\nu)=\nu /(\nu+2)$ (for $\left.z(s)=s^{2}\right)$. It is easily verified that $f$ and $g$ satisfy the hypotheses of the corollary, so $\overline{A_{m}}=C[0,1]$.

\section{REFERENCES}

1. P. Billingsley, Convergence of probability measures, Wiley, New York, 1968.

2. N. Dunford and J. T. Schwartz, Linear operators, Part I: General theory, Wiley, New York, 1988.

3. A. Friedman, Foundations of modern analysis, Dover, New York, 1970.

4. R. P. McAfee, J. McMillan, and P. Reny, Extracting the surplus in common value auctions, Econometrica 57 (1989), 1451-1459.

5. R. P. McAfee and P. Reny, Correlated information and mechanism design, Econometrica (to appear).

6. H. L. Royden, Real analysis, 2nd ed., MacMillan, New York, 1968.

Department of Economics, University of Texas, Austin, TeXas, 78712

Department of Economics, University of Western Ontario, London, Ontario N6A 5C2, CANADA 